nen gab es bei keinem Patienten; kein Todesfall wurde mit der Studientherapie in Zusammenhang gebracht.

Unter Naldemedin waren außerdem keine Anzeichen eines Opioidentzugs festzustellen. Auch die analgetische Wirkung der Opioidtherapie wurde nicht beeinträchtigt.
Fazit: Mit der einmal täglichen Gabe des oralen PAMORA Naldemedin konnte in einer zweiwöchigen Phase-III-Studie die OIC bei Patienten mit Tumorschmerzen wirksam behandelt werden. Das Medikament wurde insgesamt gut vertragen, Nebenwirkungen betrafen vor allem den Gastrointestinaltrakt; auf die Opioid- analgesie hatte die Behandlung keinen Einfluss.

Dr. Beate Schumacher

Katakami N et al. Randomized Phase III and Ex tension Studies of Naldemedine in Patients With Opioid-Induced Constipation and Cancer. J Clin Oncol 2017; 35:3859-66

\section{Massage bei Rückenschmerzen: Per Video dabei zuschauen steigert Analgesieeffekt}

Die Idee, mit der Kraft der Visualisierung zu heilen, ist nicht neu. In einer kleinen Studie wurde dieses Prinzip bei Patienten mit chronischen Rückenschmerzen untersucht, die zusätzlich zur Massage noch Videos einer Rückenmassage anschauten.
W erden schmerzende Körperstellen mit der Hand berührt und manipuliert und können die Probanden dabei direkt zuschauen, stellt sich im Vergleich zum Betrachten eines neutralen Films ein kleiner, aber signifikanter zusätzlicher analgetischer Effekt ein, so das Ergebnis mehrerer Experimentalstudien. Diese Beobachtung könnte man sich klinisch zunutze machen, indem man beispielsweise Patienten mit chronischen Rückenschmerzen per Video bei ihrer
Rückenmassage zugucken lässt. In einer kleinen Untersuchung mit 20 Patienten wurde ein möglicher Zusatzeffekt kontrolliert überprüft.

An fünf aufeinander folgenden Tagen erhielten die Rückenschmerzpatienten eine jeweils 20-minütige Massage und sahen zusätzlich in randomisierter Reihenfolge entweder eine Echtzeitvideoübertragung ihres gerade massierten Rückens sowie als Kontrollstimuli das Standbild ihres Rückens, das Bild eines

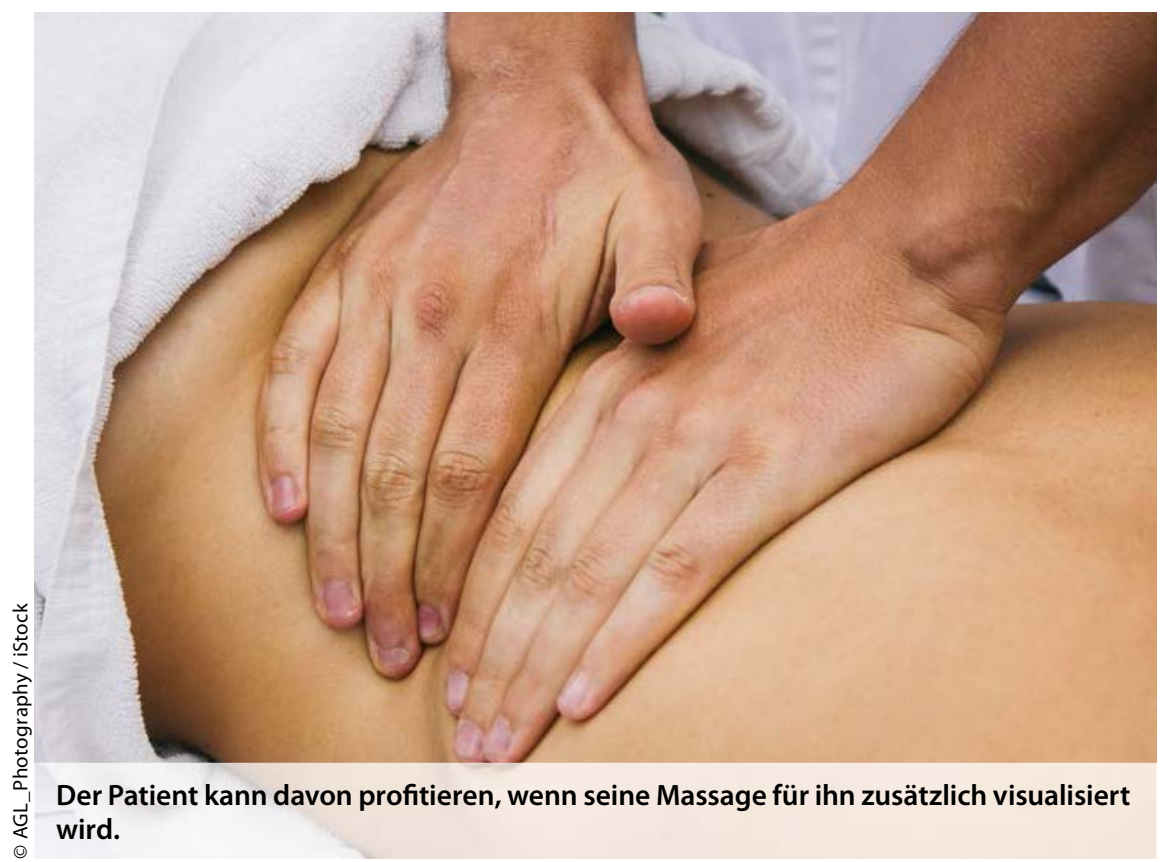

neutralen Objekts (Buch) sowie ein Video mit der Massage einer Person gleichen Geschlechts oder sie sollten einfach nur die Augen geschlossen halten.

Nach allen Sitzungen berichteten die Patienten über eine signifikante $\mathrm{Ab}$ nahme ihrer Schmerzintensität und Schmerzempfindung, jeweils gemessen mithilfe einer numerischen Ratingskala (NRS) mit den Abstufungen 0 bis 10. Das zusätzliche Betrachten des Echtzeitvideos reduzierte die Schmerzen am deutlichsten und erzielte signifikante zusätzliche Effekte im Vergleich zum Betrachten eines neutralen Objekts. Beim Vergleich mit den anderen Begleitmodalitäten zeigte sich allerdings kein signifikanter Unterschied mehr. Möglicherweise führt auch das Betrachten der Massage einer anderen Person oder die innere Vorstellungskraft bei geschlossenen Augen zur eigenen kleinen analgetischen Wirkungen, mutmaßen die Autoren. Als Erklärung für diese Beobachtung führen sie das Konzept des „Body ownership“ („Körperbesitz“) beziehungsweise eine veränderte Körperwahrnehmung im Sinne des RubberHand-Experiments von Botvinick und Cohen an. Auf die Schmerzempfindung (mehr oder weniger unangenehm) hatte das visuelle Feedback während der Massage dagegen keinen Einfluss.

Fazit: Wird Patienten mit chronischen Rückenschmerzen zusätzlich zu einer Massage noch eine visuelle Information zur eigenen aber auch der Massage einer anderen Person geboten, kann das den analgetischen Effekt weiter steigern.

Dr. Barbara Kreutzkamp

Löffler A et al. Visually induced analgesia during massage treatment in chronic back pain patients. EJP 2017; 21:1623-31 